\title{
Why simple stellar population models do not reproduce the colours of Galactic open clusters
}

\author{
A. E. Piskunov ${ }^{1,2,3}$, N. V. Kharchenko ${ }^{1,3,4}$, E. Schilbach ${ }^{3}$, S. Röser ${ }^{3}$, R.-D. Scholz ${ }^{1}$, and H. Zinnecker ${ }^{1}$ \\ 1 Astrophysikalisches Institut Potsdam, An der Sternwarte 16, 14482 Potsdam, Germany \\ email: rdscholz@aip.de \\ 2 Institute of Astronomy of the Russian Acad. Sci., 48 Pyatnitskaya Str., 109017 Moscow, Russia \\ 3 Astronomisches Rechen-Institut, Mönchhofstraße 12-14, Zentrum für Astronomie der Universität Heidelberg, \\ 69120 Heidelberg, Germany \\ 4 Main Astronomical Observatory, 27 Academica Zabolotnogo Str., 03680 Kiev, Ukraine
}

Received 9 September 2009 / Accepted 3 October 2009

\section{ABSTRACT}

\begin{abstract}
Context. For Galactic open clusters, fundamental parameters such as age or reddening are usually determined independently of their integrated colours. For extragalactic clusters, on the other hand, they are derived by comparing their integrated colours with predictions of simple stellar population (SSP) models.

Aims. We search for an explanation of the disagreement between the observed integrated colours of 650 local Galactic clusters and the theoretical colours of present-day SSP models.

Methods. We check the hypothesis that the systematic offsets between observed and theoretical colours, which are $(B-V) \approx 0.3$ and $\left(J-K_{\mathrm{s}}\right) \approx 0.8$, are caused by neglecting the discrete nature of the underlying mass function. Using Monte Carlo simulations, we construct artificial clusters of coeval stars taken from a mass distribution defined by an Salpeter initial mass function (IMF) and compare them with corresponding "continuous-IMF" SSP models.

Results. If the discreteness of the IMF is taken into account, the model fits the observations perfectly and is able to explain naturally a number of red "outliers" observed in the empirical colour-age relation. We find that the systematic offset between the continuous- and discrete-IMF colours reaches its maximum of about 0.5 in $(B-V)$ for a cluster mass $M_{\mathrm{c}}=10^{2} M_{\odot}$ at ages $\log t \approx 7$, and diminishes substantially but not completely to about one hundredth of a magnitude at $\log t>7.9$ at cluster masses $M_{\mathrm{c}}>10^{5} M_{\odot}$. At younger ages, it is still present even in massive clusters, and for $M_{\mathrm{c}} \leqslant 10^{4} M_{\odot}$ it is larger than $0.1 \mathrm{mag}$ in $(B-V)$. Only for very massive clusters $\left(M_{\mathrm{c}}>10^{6} M_{\odot}\right)$ with ages $\log t<7.5$ is the offset small (of the order of $0.04 \mathrm{mag}$ ) and smaller than the typical observational error of colours of extragalactic clusters.
\end{abstract}

Key words. open clusters and associations: general - Galaxy: stellar content - galaxies: fundamental parameters galaxies: photometry - galaxies: starburst - galaxies: star clusters

\section{Introduction}

Using data on accurate and homogeneous spatio-kinematicphotometric membership for 650 Galactic open clusters, we previously computed their integrated magnitudes in $B, V, J, H$, and

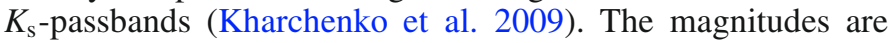
based on accurate and uniform data from the ASCC-2.5 catalogue and were computed by adding the individual luminosities of the most secure cluster members. In contrast to previous lists of integrated magnitudes (e.g., those of Battinelli et al. 1994; Lata et al. 2002) of Galactic star clusters, our data provide an independent and more importantly uniform dataset. Hence, our integrated magnitudes can and should be used as benchmarks for studies of the populations of extragalactic star clusters.

Based on these uniform data, we found disagreement between the observed colours of our cluster sample and theoretical colours derived from simple stellar population (SSP) models. The comparison of the Kharchenko et al. (2009) data with present-day SSP models such as GALEV (Anders \& Fritze-v. Alvensleben 2003) and Starburst99 (Vázquez \& Leitherer 2005) illustrated a substantial offset of the order of 0.2-0.3 mag in $(B-V)$ of the model colours with respect to the observations for clusters younger than $\log t \lesssim 8.5$. This is both remarkable and important, since most data such as age and mass collected nowadays for the bulk of extragalactic clusters, are derived by comparing their integrated light with the predictions of SSP models (see e.g., Bik et al. 2003). The models are produced by evolutionary synthesis codes simulating the extensive and complicated stellar populations of galaxies. However, they are also believed to be appropriate for describing cluster populations more than five orders of magnitude less massive (and apparently far simpler). Although star clusters may be influenced by effects that are irrelevant to galaxies (e.g., low number statistics, because of the discreteness of the real IMF, or dynamical mass-loss by evaporation of stars), the validity of the SSP approach for star clusters can be tested from general considerations (Cerviño \& Luridiana 2004). In contrast, in this paper we apply the issue of the discreteness of the IMF to the specific case of Galactic open clusters.

A colour offset of this kind was found previously by Lata et al. (2002), in the colour indices $U-B, B-V, V-R$, and $V-I$. However, they accept that only the offsets in $V-R$ and $V-I$ represent significant deviation of their observations from the model predictions. We suspect that the discreteness of the IMF in open 
clusters may explain these discrepancies. To study this issue in a more systematic way, we constructed our own SSP model that takes into account the effect of low number statistics (discreteness), which is a common problem for open star clusters. We cross-checked a continuous version of our code with GALEV and found good agreement.

The discreteness of the IMF itself is a natural assumption for any stellar ensemble consisting of individual objects. However, when one considers vast stellar populations (e.g., galaxies) that densely populate the entire colour-magnitude diagram, one can adopt a continuous IMF, which is more convenient for different technical reasons. For small populations (stellar clusters), the assumption of a discrete IMF is clearly appropriate. This letter describes the solution of a restricted problem, namely the influence of the discrete-IMF approach on the colours of young clusters. The full scope of results related to the IMF-discreteness effect on star clusters will be reported elsewhere.

\section{The SSP models and observations}

For the determination of integrated magnitudes (and colours) of Galactic open clusters, we refer the reader to Kharchenko et al. (2009), which also gives an overview of the cluster sample and references for further reading.

For simplicity, we refer to our SSP model hereafter as the "realistic" model, and call the literature models "standard" models. The standard SSP models were computed with help of the online servers provided by the GALEV ${ }^{1}$, and Starburst $99^{2}$ sites.

The input parameters of the realistic model are the total cluster mass $M_{\mathrm{c}}$, the initial mass function (IMF) of cluster stars

$f(m)=\mathrm{d} N / \mathrm{d} m$,

( $N$ being the number of stars with masses between $m, m+\mathrm{d} m$ ), and the lower and upper limits $\left(m_{1}\right.$ and $\left.m_{\mathrm{u}}\right)$ of the range of the allowed masses of cluster stars. We adopt the Salpeter IMF, $f(m)=k m^{-\alpha}, \alpha=2.35$, where $k$ is a normalisation factor that depends on the assumed cluster mass. The upper mass limit is confined to the high-mass end of the adopted grid of evolutionary tracks/isochrones. Only at the very early stages of cluster evolution does $m_{\mathrm{u}}$ have an impact on integrated colours. Given a fixed IMF slope, the lower limit governs the population of the massive end of the IMF at given $M_{\mathrm{c}}$. We consider $M_{\mathrm{c}}$ and $m_{\mathrm{l}}$ here as free parameters of the adopted model. The model stellar sample was generated from the IMF with a random number generator.

The photometric properties of the model are defined by the implemented grid of the isochrones. For our model, we have used the grid provided by the Padova group via the online server $\mathrm{CMD}^{3}$. According to our experience, the Padova isochrones fit the brighter parts of the open cluster CMDs reasonably well over a wide range of cluster ages. These parts are responsible for the integrated colours. We used a solar metallicity grid $(Z=0.019)$, and retrieved the passbands $U, B, V, R, I$ and $J, H, K_{\mathrm{s}}$ with an age range $\log t=6.0-10.2$ and a step in $\log t$ of 0.05 . For compatibility, we selected the same set of parameters (i.e., underlying IMF, mass range limits, isochrone set) for the standard models. However, since the Padova group regularly updates their isochrones, full compatibility between the isochrone grids used by the realistic and standard models cannot be guaranteed.

\footnotetext{
1 http://www.galev.org/

2 http://www.stsci.edu/science/starburst99/

${ }^{3}$ http://stev.oapd.inaf.it/cgi-bin/cmd
}
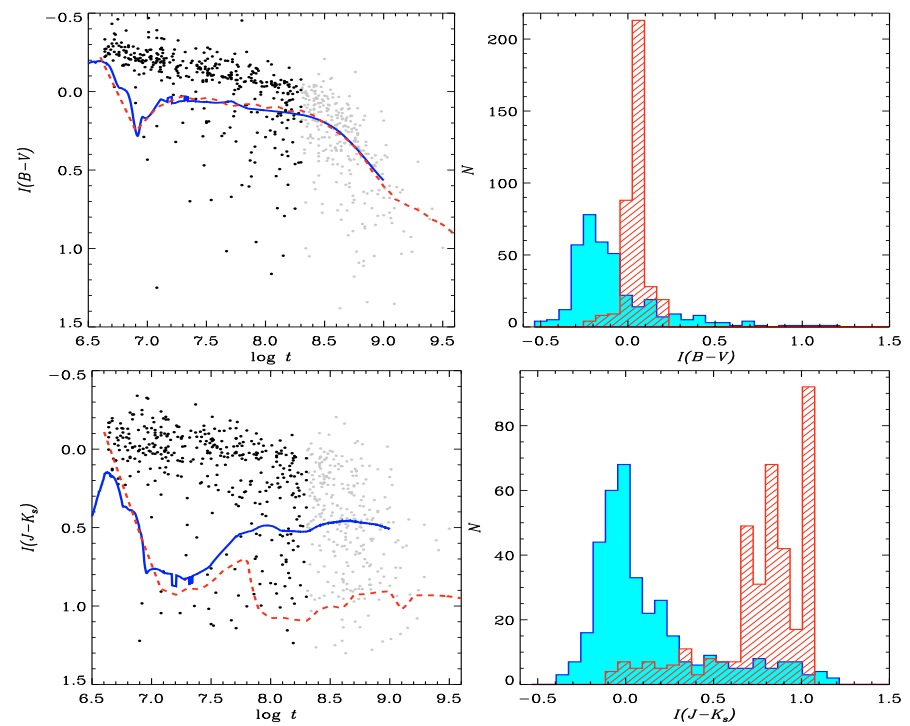

Fig. 1. Observed colours of 650 Galactic open clusters compared to theoretical colours computed from standard SSP models. The upper row is for $B-V$, the bottom one for $J-K_{\mathrm{s}}$. The left column shows "integrated colour vs. $\log t$ " diagrams, the right one the distributions of colour indices. Dots show Galactic open clusters from our sample. Black dots are young clusters used for constructing the colour distribution, grey dots the older clusters. The curves are the SSP model tracks (the solid one is from Starburst99, the dashed one is from GALEV).The observed colour distributions are shown as the filled cyan histogram, the GALEV model distribution as the hatched red one.

In Fig. 1, we compare the location of the observations with the curves of the standard model in the colour-age diagram. GALEV and Starburst99 tracks agree reasonably well with each other. The above-mentioned offset of the models with respect to observed colours for $\log t \lesssim 8.5$ is obvious in both of the colourage diagrams.

The observed clusters show a clear pattern, which at $\log t \lesssim$ 8.5 is represented by "main sequence" clusters (not containing red giants or supergiants). The considerable redward spread of observed points cannot be attributed to photometric errors; it is provided in this part of the diagram by a few clusters containing yellow or red supergiants. For $\log t>8.5$, one finds, in general, clusters with red giants. The colour spread of older clusters is caused by different evolutionary stages of red giants in clusters of different ages. The depletion of the cluster sequence after $\log t \approx 8.5$ can be explained by either cluster mortality (the typical lifetime of local clusters is only around $\log t \approx 8.5$, according to Piskunov et al. 2006) or selection effects, which are more significant for older clusters.

In the right column of Fig. 1, we compare the distributions of the observed cluster colours with the GALEV predictions. We took the ages of the real clusters from Kharchenko et al. (2009), and calculated the integrated colours from the GALEV model. The discrepancy between the observed colours and those from the standard model is obvious. The local star clusters in our Galaxy are much bluer (by about 0.3 mag in the optical and about $0.8 \mathrm{mag}$ in the near IR) than "theoretical" clusters of the same age. We discuss this below in connection with the results for the realistic model.

\section{The discreteness of the IMF and the SSP models}

As we have already mentioned, a possible explanation of the observed disagreement is the continuity of the IMF adopted in 

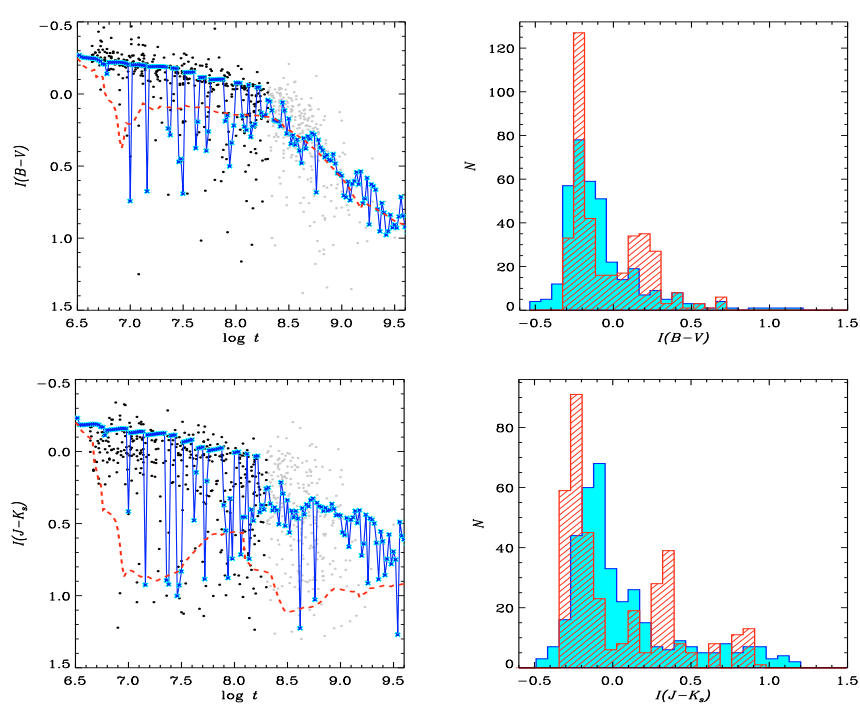

Fig. 2. Observed colours of 650 Galactic open clusters compared to theoretical colours computed from realistic and standard SSP models. The upper row is for $B-V$, the bottom one for $J-K_{\mathrm{s}}$. The left column shows "integrated colour vs. $\log t$ " diagrams, the right one the distributions of colour indices. Dots show Galactic open clusters from our sample. Black dots are young clusters used for constructing the colour distributions, grey dots the older clusters. The curves are the model tracks constructed in this paper. The dashed curve is for the standard model, crosses show the realistic one. The observed colour distributions are shown as filled cyan histograms, the realistic model distribution as the hatched red ones.

standard SSP models, which means that every small mass interval of the given isochrone emits light according to its $T_{\text {eff }}$ and $\log g$. The total flux that a "theoretical" cluster emits is a result of the integration over the isochrone, weighted by the given IMF. It is immediately obvious that luminosity then scales with mass, once the age (or the isochrone) is fixed. In the discrete approach, however, only points on the isochrone where stars of a respective mass are actually present contribute. The random realisations of the IMF have statistical fluctuations even for a smooth probability density distribution (i.e., smooth IMF). At the high-mass end of the mass range, these fluctuations are particularly large because of low number statistics, and produce gaps in the mass spectrum. In the case of a single supergiant present in the cluster at a given instant of time - a case frequently occurring in our local Galactic clusters - the colour fluctuations might be especially strong. If the main sequence lifetime of the star closest in mass to the supergiant exceeds the supergiant's lifetime, then after the supergiant's death the less-massive star remains on the main sequence, which makes the cluster considerably bluer than it was before. The sparser the stellar population of a cluster is (i.e., the lower its total mass) the stronger the colour fluctuations are in the course of its evolution. After the most massive stars die, the red giant population of a cluster reaches an equilibrium and the colour fluctuations of a cluster begin to weaken.

Figure 2 is the counterpart of Fig. 1 for a realistic model of the scenario described above. The solid line with crosses and the dashed line shown here correspond to the discrete-IMF and to the continuous-IMF regimes, respectively, of the same model. The total initial mass of the model is assumed to be $M_{\mathrm{c}}=1000 M_{\odot}$, and $m_{1}=0.08 M_{\odot}$. These parameters were chosen to provide a fit to the colour distribution in the simplest case of our local young cluster population. The ultimate upper mass limit $m_{\mathrm{u}}=100 M_{\odot}$ is defined by the Padova grid of isochrones, and $m_{\mathrm{u}}$ depends on both of the adopted $M_{\mathrm{c}}$ and $m_{1}$. For example, for $M_{\mathrm{c}}=1000 M_{\odot}$ and $m_{1}=0.01,0.1$, and $1 M_{\odot}$, the upper mass limit to the simulated mass spectrum equals $m_{\mathrm{u}} \approx 60,90$, and $100 M_{\odot}$. In the latter case, $m_{\mathrm{u}}$ is limited by the adopted evolutionary grid.

For the standard models, this choice of parameters is however not important. The derived evolutionary track depends on neither $M_{\mathrm{c}}$ nor $m_{\mathrm{l}}$. The upper mass limit affects only the early stages of the cluster evolution. The only parameter influencing the standard model is the IMF slope $\alpha$, although this influence is not strong. To change substantially the track, one would need to increase the exponent $\alpha$ to 4.35. A flattening of the IMF with respect to the Salpeter value of the slope does not have a strong effect on the colour-age relation. We note that our continuousIMF model agrees with the predictions of the GALEV code and, hence, produces colours redder than the observed colours at $\log t<8.5$.

The realistic model with the above parameters produces a mass spectrum consisting of 3532 stars. In the mass range $5 M_{\odot}>m>0.3 M_{\odot}$, however, the number of stars is only 599 . This is of the same size as the number of Pleiades members observed in this mass range, which according to Belikov et al. (1998) equals 780. Thus, the model appears to be comparable to the Pleiades. The number of model stars with $m>5 M_{\odot}$ is equal to 20. So, before the model cluster reaches the age of the Pleiades $(\log t \approx 8.1)$, it has produced 20 red supergiants. The twenty corresponding "RG-events" are clearly seen in Fig. 2. At all other times (until $\log t \approx 8.1$ ), the cluster is seen as a "mainsequence cluster" and resides in the MS-domain of the diagram. If we increase the lower bound $m_{1}$, the number of massive stars increases and the fraction of "RG-events" slowly increases, especially at older ages. But even at $m_{1}=1 M_{\odot}$, the "MS-clusters" are present in the track up to $\log t \approx 8.2$. On the other hand, decreasing $m_{1}$ diminishes the number of "RG-events". In principle, from the frequency of red outliers in the "colour versus $\log t$ " diagram, one can estimate indirectly the parameter $m_{1}$.

Although the above example provides only an explanation for the local cluster population of typical mass of order of $10^{3} M_{\odot}$, it nevertheless illustrates the effect of the discreteness on extragalactic clusters where the bulk of cluster data comes from analysing the integrated light. For clusters of mass of the order of $10^{5} M_{\odot}$ Dolphin \& Kennicutt (2002) found that this effect is rather small. This finding was interpreted by some workers (e.g., Bastian et al. 2005) as a justification for neglecting discreteness. Among other arguments, Bastian et al. (2005) refer to the large masses of the observed extragalactic clusters, and assume that the effect only produces a symmetric spread around the "true" sequence. However, observations also find extragalactic clusters with masses typical of Galactic clusters $\log M_{\mathrm{c}}=2-5$ (see Mora et al. 2009). Since Fig. 2 shows that the effect is highly asymmetric with respect to the standard SSP sequence, a study of the effect over the entire range of cluster masses is appropriate.

To estimate the dependence of the discreteness effect on cluster age and mass, we have performed a series of Monte Carlo simulations. We considered a set of cluster models with masses spread over the range $\log M_{\mathrm{c}}=2.0-6.5$. For every model of a particular mass at the starting point, the specific realisation of the initial mass spectrum was sampled at random from the IMF. The evolution of the synthetic cluster was then followed up to $\log t<8.3$. At every time step, we compared the colours of the realistic model with its standard counterpart and computed the colour differences $\delta=I(B-V)_{\mathrm{r}}-I(B-V)_{\mathrm{s}}$. Here the subscript $r$ represents "realistic", and $s$ "standard". For every $M_{\mathrm{c}}$, 

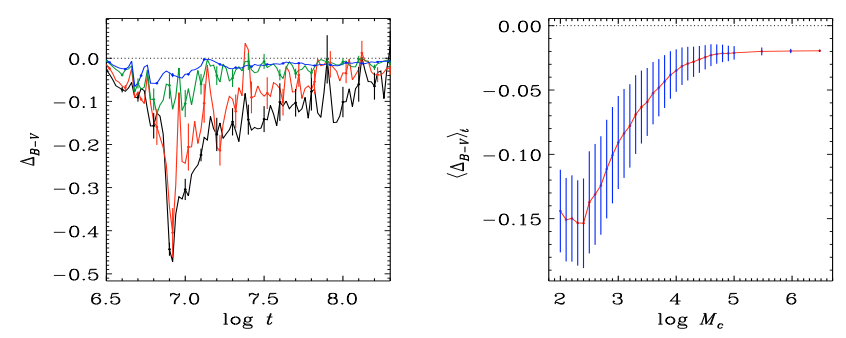

Fig. 3. The colour displacement versus age relations (left) for cluster models of different masses. The curves correspond (from bottom to top) to $\log M_{\mathrm{c}}=2,3,4,6.5$. The vertical bars indicate the statistical uncertainty $\varepsilon$. In the right panel, we show the displacement averaged over the age interval $\log t=6.5-8.3$ as a function of cluster mass. The bars indicate the error in the averaging.

we compiled $N=50$ evolutionary sequences of cluster models by considering different statistical realisations of the initial mass spectrum, and evaluated at every time step the corresponding average difference in colour

$\Delta=\sum_{i=1}^{N} \delta_{i} / N$,

and its statistical uncertainty $\varepsilon$ computed to be

$\varepsilon^{2}=\sum_{i=1}^{N}\left(\delta_{i}-\Delta\right)^{2} /(N-1) / N$.

In Fig. 3, we summarize the colour bias produced by neglecting the IMF-discreteness effect, in the dependence on both the age and mass of a star cluster. The left panel shows the dependence of $\Delta$ on cluster age. The curves are for different model cluster masses. The bars indicate the uncertainty $\varepsilon$. The first conclusion from Fig. 3 is that there is an overall negative bias. As expected, the maximum effect is observed at $\log t \approx 7$, when the first red stars appear in the upper, sparsely populated CMD of a cluster. At younger ages, the effect diminishes since both realistic and standard models are populated with main-sequence stars only, which have approximately the same colour indices at the upper MS. At $\log t>7$, the effect slowly weakens, reflecting the CMD geometry (distance between the MS and Hayashi limit). At $\log t \approx 8,|\Delta|$ becomes lower than 0.1 for all considered masses. With increasing cluster mass, the effect in general diminishes. It is interesting to note that even at $\log M_{\mathrm{c}}=6.5$ the effect is small, but significant, and at $\log t=6.7-7.0$ the colour shift is of the order of a few hundredths of a magnitude. For masses $\log M_{\mathrm{c}}>4$, $|\Delta|$ remains below 0.1 at all ages considered.

The explicit dependence of the discreteness effect on cluster mass, providing the net-estimate of the effect for young clusters, can be derived if one averages the $\Delta$ versus $\log t$ relations over the entire range of ages. The result is shown in the right panel of Fig. 3. The effect is considerable $\left(\left|\langle\Delta\rangle_{t}\right| \gtrsim 0.05\right)$ for $\log M_{\mathrm{c}}<4$. At higher masses it is small although significant.

For the other colours the behaviour is qualitatively similar, although the redder the passband, the stronger the effect is. This is intuitively understandable. The effect is caused by quantization, because red stars radiate proportionally more of their light at longer wavelengths. For example, in the case of $I\left(J-K_{\mathrm{s}}\right)$ the largest difference between the realistic and the standard models is about -0.9 (cf. Fig. 3). In a forthcoming paper, this issue will be discussed in more detail.

\section{Concluding remarks}

Observations of local Galactic open clusters have enabled us to measure their integrated magnitudes, masses, ages, and reddening. Using this data set, we have found a remarkable discrepancy between the observed colours and the predictions of SSP models. The main reason for this disagreement is the neglect of the assumption of IMF-discreteness. When this effect is taken into account, the model agrees adequately with the observations and is even able to explain the large colour spread observed in the empirical colour-age relation in a natural way.

In which conditions is the effect of discreteness relevant? Since it is a consequence of low-number statistics, it depends on the sparseness of the stellar population in the upper CMD of a cluster where the bulk of light is emitted. The density of the population depends primarily on cluster mass, and in addition on cluster age, on [strange at first glance] the lower mass limit of the stars formed, and to some degree on the slope of the IMF. In the context of this letter, other factors are not important.

According to our investigation, the systematic offset between the continuous- and discrete-IMF colours diminishes substantially but not completely at $\log t>7.9$, at cluster masses $M_{\mathrm{c}}>$ $10^{5} M_{\odot}$. At younger ages, it remains present even in massive clusters, and for $M_{\mathrm{c}} \leqslant 10^{4}$ it is larger than $0.1 \mathrm{mag}$ in $(B-V)$. Only for very massive clusters $\left(M_{\mathrm{c}}>10^{6} M_{\odot}\right)$ and young ages $(\log t<7.5)$, the offset falls below a typical observational error. We note in passing that the effect is stronger for redder passbands. These findings are in good agreement with the theoretical forecast of Cerviño \& Luridiana (2004). The immediate consequence of the application of a continuous-IMF approach to the SSP models of stellar clusters is a systematic underestimate of reddening. Other problems affect the age and mass determination based on evolutionary grids of these models (at least for masses below $10^{6} M_{\odot}$ ). Because of the rather flat colourage relation of young clusters, a systematic error of the order of 0.1 mag produces an error in cluster age of about one order of magnitude. On the other hand, there is a danger that the existence of a large number of "main-sequence" (i.e., blue) clusters could be interpreted as evidence of a recent burst of star formation. We can exclude such a burst having occured in our Galactic open clusters even if they are blue. Finally, the technique of the parameter determination should be changed to incorporate the cluster flux variations caused by the discrete nature of the upper IMF.

Acknowledgements. This study was supported by DFG grant 436 RUS 113/757/0-2, and RFBR grant 07-02-91566. We thank Drs. Anders and Maíz Apellániz for drawing our attention to the Cerviño \& Luridiana paper.

\section{References}

Anders, P., \& Fritze-v. Alvensleben, U. 2003, A\&A, 401, 1063

Bastian, N., Gieles, M., Lamers, H. J. G. L. M., Scheepmaker, R. A., \& de Grijs, R. 2005, A\&A, 431, 905

Battinelli, P., Brandimarti, A., \& Capuzzo-Dolcetta, R. 1994, A\&AS, 104, 379 Belikov, A. N., Hirte, S., Meusinger, H., Piskunov, A. E., \& Schilbach, E. 1998, A\&A, 332, 575

Bik, A., Lamers, H. J. G. L. M., Bastian, N., Panagia, N., \& Romaniello, M. 2003, A\&A, 397, 473

Cerviño, M., \& Luridiana, V. 2004, A\&A, 413, 145

Dolphin, A. E., \& Kennicutt, Jr., R. C. 2002, AJ, 124, 158

Kharchenko, N. V., Piskunov, A. E., Röser, S., et al. 2009, A\&A, 504, 681

Lata, S., Pandey, A. K., Sagar, R., et al. 2002, A\&A, 388, 158

Mora, M. D., Larsen, S. S., Kissler-Patig, M., Brodie, J. P., \& Richtler, T. 2009, A\&A, 501, 949

Piskunov, A. E., Kharchenko, N. V., Röser, S., Schilbach, E., \& Scholz, R.-D. 2006, A\&A, 445, 545

Vázquez, G. A., \& Leitherer, C. 2005, ApJ, 621, 695 